\title{
Mortalidad por COVID-19 asociada a comorbilidades en pacientes del Instituto Salvadoreño del Seguro Social
}

\author{
DOI 10.5377/alerta.v4i2.10366 \\ Víctor David Franco ${ }^{1}$, Luís Morales Chorro ${ }^{2}$, Rafael Baltrons Orellana ${ }^{3}$, Carlos Romeo Rodríguez Salmerón ${ }^{4}$, \\ Oscar Santos Urbina ${ }^{5}$, Claudia López de Blanco ${ }^{6}$ \\ 1. Departamento de investigación y docencia en salud, Instituto Salvadoreño del Seguro Social, San Salvador, El Salvador. \\ 2. Departamento de epidemiología, Instituto Salvadoreño del Seguro Social, San Salvador, El Salvador. \\ 3. Departamento de epidemiología, Hospital Amatepec, Instituto Salvadoreño del Seguro Social, San Salvador, El Salvador. \\ 4. Hospital Amatepec, Instituto Salvadoreño del Seguro Social, San Salvador, El Salvador. \\ 5. Departamento de medicina interna, Hospital Regional, San Miguel, El Salvador. \\ 6. Departamento de investigación y docencia en salud, Instituto Salvadoreño del Seguro Social, San Salvador, El Salvador. \\ ${ }^{*}$ Correspondencia \\ 】victor.franco@isss.gob.sv
}

1. (1) 0000-0003-4087-838X

\section{ACCESO ABIERTO}

Mortality due to COVID-19 associated with comorbidities in patients of the Salvadoran Social Security Institute

Citación recomendada: Franco VD, Morales Chorro L, Baltrons Orellana R, Rodríguez Salmerón CR, Urbina O, López de Blanco C. Mortalidad por COVID-19 asociada a comorbilidades en pacientes del Instituto Salvadoreño del Seguro Social. Alerta. 2021;4(2): 28-37. DOI:10.5377/alerta. v4i2. 10366

Recibido:

16 de noviembre 2020

Aceptado:

14 de mayo 2021

Publicado:

21 de mayo 2021

Contribución de autoría:

VDF1. Concepción,

recolección, diseño, análisis e interpretación de datos

y redacción del artículo.

LMC2, RBO3, CRRS4 y OSU5.

Recopilación y envío de

datos, revisión del articulo y

aprobación de la versión final.

CLB6. Asesoría metodológica

y del comité de ética

institucional.

\section{Conflicto de intereses:}

Los autores declaran no tener

conflictos de interés en el

estudio.

\section{Resumen}

Introducción. La infección respiratoria por coronavirus ha generado gran cantidad de muertes desde su inicio en China en diciembre 2019. Debido a que es una enfermedad desconocida, son necesarios estudios para mejorar el abordaje, especialmente a personas en riesgo. Objetivo. Establecer cuál fue la supervivencia y riesgo de muerte observada en pacientes del Instituto Salvadoreño del Seguro Social con diagnóstico o sospecha de COVID-19 y factores de riesgo asociados. Metodología. Estudio transversal analítico de 2,670 pacientes contagiados (o posibles). Las variables fueron analizadas con el método de Kaplan Meier y el modelo proporcional de riesgo de Cox. Resultados. Los no sobrevivientes incluyeron personas mayores y con enfermedades subyacentes como hipertensión, diabetes, insuficiencia renal y cáncer. La hipertensión arterial presentó un hazard ratio e intervalo de confianza 95 \% de 2,58/2,3-2,9 y la diabetes, 2,56/2,2-2,9. La edad $<60$ años $(0,34 / 0,30-0,39)$ y la ausencia de enfermedades $(0,31 / 0,27-0,35)$ se asocian a lo contrario. La supervivencia general fue $93,6 \% / 92,5-94,5 \%$ al día 1 , y del 50,4\%/48,1-52,6\% a 15 días. Las enfermedades cardíacas generaron la menor supervivencia a 5 días $(71,4 \%$ a 41,2 \%) y el cáncer a 15 días (51,1 \% a 3,9\%). Conclusión. Las personas menores de 60 años sin comorbilidades tienen menor riesgo de muerte comparadas con las que tienen comorbilidades como diabetes, hipertensión y cáncer que presentaron un riesgo 3 veces mayor de muerte intrahospitalaria por COVID-19 y menor supervivencia a 15 días.

Palabras clave

COVID-19, mortalidad, comorbilidad, supervivencia

\section{Abstract}

Introduction. The respiratory infection by coronavirus has generated a large number of deaths since its beginning in China in December 2019. Because it is an unknown disease, studies are needed to improve the approach specially to people at risk. Objective. Investigate factors associated with higher fatalities from COVID-19 in patients from the Salvadoran Social Security Institute. Methodology. Cross-sectional analysis of 2,670 infected (or possible) patients. The variables were analyzed with the Kaplan Meier method and the Cox proportional risk model. Results. Cohort verified between April and August 2020, non-survivors included older people and with underlying diseases such as hypertension, diabetes, kidney failure and cancer. Hypertension had a hazard ratio and $95 \%$ confidence interval of 2,58/2,3-2,9 and diabetes, 2,56/2,2-2,9. Age under $60(0,34 / 0,30-0,39)$ and absence of disease $(0,31 / 0,27-0,35)$ are associated with the opposite. Overall survival was $93,6 \% / 92,5-94,5 \%$ at day 1 , and $50,4 \% / 48,1-52,6 \%$ at day 15 th. Heart disease had the lowest 5 -day survival $(71,4 \%$ to $41,2 \%)$ and cancer at 15 days $(51,1 \%$ to $3,9 \%)$. Conclusion. People under 60 without comorbidities have a lower risk of death compared to those with comorbidities such as diabetes, hypertension and cancer, which presented a 3 times higher risk of in-hospital death from COVID-19 and lower survival at 15 days.

Keywords

COVID-19, mortality, comorbidity, survival 


\section{Introducción}

La pandemia de influenza de 1918 fue causada por un virus $\mathrm{H} 1 \mathrm{~N} 1$ que genéticamente estaba relacionado con las aves. Entre 1918 y 1919 esta enfermedad se diseminó por todo el planeta y se calcula que infectó a más de 500 millones de personas, matando al menos a 50 millones en todo el mundo'.

Los primeros casos de COVID-19 fueron reportados en diciembre del 2019 en Wuhan, provincia de Hubei en China². Veintisiete personas con neumonía grave fueron diagnosticadas y reportadas por la Organización Mundial de la Salud ${ }^{3}$. Posteriormente se dio a conocer que el agente causal es el virus de la familia coronavirideae al cual se llamó virus del síndrome respiratorio agudo severo tipo-2 (SARS-CoV-2) o coronavirus-20194.

Estos virus se catalogan en coronavirus adquiridos en la comunidad y en coronavirus zoonóticos. Estos últimos circulan transitoriamente, pero generan grandes epidemias de enfermedad respiratoria grave, otros virus del mismo grupo son el coronavirus del síndrome respiratorio agudo severo (SARS), identificado por primera vez en Guangdong, China, en noviembre del 2002 y el coronavirus del síndrome respiratorio del Medio Oriente (MERS), cuyo primer brote fue reportado en Zarga, Jordania, en abril del $2012^{4}$.

El agente en cuestión es un virus RNA de cadena sencilla, esférico, con un diámetro de $125 \mathrm{~nm}$, polaridad positiva y longitud de 30,000 ribonucleótidos ${ }^{5}$. Este virus tiene un periodo de incubación de 5,2 días (rango de 2 a 14) e ingresa a la célula utilizando los receptores de la enzima convertidora de angiotensina 2 (ACE2) 6 .

La infección se ha diseminado globalmente. Para abril del 2020 se registraban más de 2,6 millones de casos con una mortalidad no vista desde la pandemia de gripe española en el primer decenio del siglo XX? Según datos oficiales del Ministerio de Salud, el primer caso sospechoso se reportó el mismo mes en que la COVID-19 se declaró pandemia ${ }^{8}$.

Este primer caso era proveniente del extranjero y al cierre de esta investigación (31 agosto 2020) ya se totalizaban más de 100 casos «importados» (personas que arribaron al país con la enfermedad en periodo de incubación o sin manifestaciones clínicas confirmadas en centros de cuarentena) y 2,700 personas infectadas, denominadas «varados» (salvadoreños enfermos en el extranjero a los cuales no se le permitía el ingreso al país $)^{9}$.
La situación nacional de la pandemia en esa fecha fue de 25,700 casos confirmados; de estas, 14,292 personas han superado la enfermedad con éxito y las personas que no sobrevivieron superan los 710 casos. El culmen de la pandemia se observó entre julio y agosto del 2020, tiempo en el cual se llegaron a diagnosticar más de 450 casos en un solo día?.

La COVID-19 ha afectado más a los hombres con edades entre los 20 a 39 años, la mayoría han cursado sin mayor sintomatología y el nivel de compromiso general ha sido de leve a moderado?. Además, se ha observado una incidencia en niños (0 a 9 años) considerablemente menor que en adultos $^{10}$.

Debido a que esta es una enfermedad nueva, es necesario realizar análisis que ayuden a identificar las personas que sean proclives a un pronóstico más sombrío, de tal forma que, cuando se intervengan personas sospechosas de COVID-19 y presenten alguna condición de las que han sido identificadas como de mal pronóstico, sean manejados con mayor detenimiento más rápida y agresivamente.

Este estudio busca establecer cuál fue la supervivencia y riesgo de muerte observada en las personas con diagnóstico o sospecha de COVID-19 y además factores de riesgo, como la edad, sexo, presencia de comorbilidades y condiciones de salud (obesidad, secuelas de accidente cerebro vascular, patología tiroidea, diabetes mellitus, hipertensión arterial, insuficiencia renal crónica, cirrosis hepática, cáncer, cardiopatías isquémicas y neumopatías crónicas).

\section{Metodología}

El diseño de este estudio es transversal analítico y se realizó a partir de la información disponible en los registros oficiales del departamento de actuariado y estadística del Instituto Salvadoreño del Seguro Social (ISSS) sobre los movimientos hospitalarios de pacientes 3 hospitales del ISSS destinados al manejo exclusivo de pacientes con COVID-19 (hospital general, hospital Amatepec y hospital regional de San Miguel) entre abril y agosto 2020 .

Luego de una revisión inicial detallada de los registros de hospitalización, se localizaron 2512 casos que fueron atendidos en los servicios de hospitalización, a estos se sumaron 1,298 casos provenientes de áreas que fueron adecuadas para la atención de pacientes con COVID-19 (observación, máxima respiratoria, griparios y hospitales de día); de un total de 3810 casos, se eliminaron 952 registros repetidos (varias 
consultas del mismo paciente). Se inició la revisión con 2858 casos, de los cuales se descartaron otros 126 por presentar errores en la afiliación y vacíos de información que imposibilitaron el seguimiento y otros 62 casos más por tratarse de pacientes a los que se les descartó que fueran casos de COVID-19 (gripe común, fiebre tifoidea, síndrome diarreico agudo entre otros), finalmente quedó un total de 2670 casos con los cuales se realizó el estudio.

La información clínica fue complementada con otros registros sobre asistencias hospitalarias anteriores (desde enero de 2018 a junio 2020). Estos datos también fueron obtenidos de departamento de actuariado y estadística del ISSS y de los registros de producción de consulta externa de los centros de manejo ambulatorio con expediente electrónico (consultorio de especialidades, consulta externa del hospital general, unidad médica de Santa Ana y las clínicas comunales Las Victorias, Mejicanos, Ayutuxtepeque, Miramonte y San Antonio Abad).

Se tabuló la información sobre afiliación, edad, sexo, fecha de ingreso, de egreso, condición al alta, antecedentes médicos como diabetes mellitus, insuficiencia renal crónica, hipertensión arterial, cáncer, enfermedades cardíacas y pulmonares crónicas, colagenopatías y enfermedades del tiroides entre otras. La presencia o no de dichos antecedentes médicos fue dicotomizada como «0» ausencia y «1» presencia. Toda la información se volcó en una hoja de cálculo de Excel 2016, donde se llevó a cabo su procesamiento inicial. Las variables continuas se presentan en promedios y desviación estándar y las categóricas en valores absolutos. Estas últimas fueron comparadas entre pacientes que presentaban o no las condiciones de interés para el estudio y entre sobrevivientes y no sobrevivientes.

Se empleó el modelo proporcional de riesgo de Cox para determinar la relación de las condiciones clínicas y patológicas con mayor mortalidad por COVID-19. La prueba de Mantel-Cox (prueba de logaritmo de rango) se usó para establecer si la diferencia encontrada poseía significancia estadística. Se elaboraron tablas de vida para calcular la supervivencia estimada a los días 1, 5, 10 y 15 posterior a su ingreso en el hospital y según presentaron o no las condiciones de interés. También se presenta la información sobre supervivencia en gráficas de Kaplan Meier, los análisis fueron realizados con el programa STATA versión 14,0 (STATA Corp., College Station, TX, USA) y para todos se consideró estadísticamente válido un valor de $p \leq 0,05$ y los intervalos de confianza se establecie- ron al $95 \%$. La investigación cuenta con aprobación del comité de ética del ISSS.

\section{Resultados}

La información sobre las características clínico-epidemiológicas de los pacientes se presenta en la Tabla 1. La edad promedio fue 60 años con desviación estándar de 16,3. De los 2670 casos, 68,4 \% son hombres, 38,5 $\%(1,028)$ tienen entre 40 a 59 años, 37,9 $\%(1,013)$ entre 60 a 79 y 10,9 \% (293) más de 80 años. El 65,3\% (1743) son casos con diagnóstico no confirmado. El $64,6 \%$ de pacientes no tiene historia de haber presentado alguna enfermedad crónica, 51,1\% tienen edades inferiores al promedio. La distribución de las comorbilidades de mayor frecuencia fue 17,0 \% para hipertensión arterial, diabetes mellitus $14,6 \%$, insuficiencia renal crónica 6,10 \%, obesidad 4,7\% y 2,7\% para las cardiopatías y trastornos del ritmo.

Se registraron 1,234 muertes (46,2\%), el $87 \%$ se presentaron en pacientes que se encontraban en los servicios de hospitalización, el 69,8\% fueron hombres, el 50,7 \% (626) con edades entre 40 a 59 años. La población que falleció y que concomitantemente adolecían de otras condiciones crónicas de salud se distribuyeron así: hipertensión arterial 28,5 \% (352) y diabetes mellitus 24,6 \% (304). La mortalidad específica más alta, según condición preexistente, se dio en aquellos pacientes con cirrosis hepática de los cuales fallecieron 23 de los 25 cirróticos detectados en el escrutinio (92\%), seguidos de los pacientes con cáncer $(81,8 \%)$, diabéticos $(77,7 \%)$, hipertensos (77,4\%), cardiópatas crónicos (76 \%) y enfermos renales (71,8\%).

Las condiciones de salud presentes que modificaron la probabilidad de fallecer al contagiarse con COVID-19 en estas personas, se presentan la Figura 1. Las relacionadas a una mayor mortalidad fueron hipertensión arterial con hazard ratio (HR) de 2,58 e intervalo de confianza del $95 \%$ entre 2,26-2,95 y un valor de $p<0.05$, seguido de presentar 2 de estas enfermedades crónicas en simultaneo HR 2,57 e IC95\% 2,20-3,0, p $<0,05$, diabetes mellitus 2,56 y 2,23 - 2,93 con p <0,05, cardiopatías crónicas 2,53 y $1,84-3,48 p<0,05$, neoplasias 2,44 y $1,72-$ $3,47 p<0,05$. La ausencia de enfermedad crónica se encontró con un efecto contrario, HR: 0,31 e IC95 \%: 0,27- 0,35, $p<0,05$, edad $<60$ años 0,34 y 0,30 - 0,39, $p<0,05$. En esta misma figura se presentan los resultados de la prueba de Mantel-Cox, donde se definen las diferencias con mayor significancia estadística por prueba del logaritmo de rango (Log Rank). 
Tabla 1. Condiciones clínico-epidemiológicas y de mortalidad en pacientes con infección respiratoria aguda por COVID-19 comprobada o por sospecha clínica $(\mathrm{N}=2,670)$.

\begin{tabular}{|c|c|c|c|}
\hline Variable & Casos & $\%$ & Observaciones \\
\hline Sexo masculino & 1,826 & 68,4 & - \\
\hline Edad promedio & 60 & - & DS:16.3, Min:15, Max: 99 \\
\hline Casos sospechosos (U072) & 1,743 & 65,3 & - \\
\hline Pacientes hospitalizados & 2,510 & 94,0 & - \\
\hline
\end{tabular}

\begin{tabular}{lrr}
\hline Distribución por grupos de edad & & \\
\hline$\leq 20$ a 39 & 336 & $12,6-$ \\
40 a 59 & 1,028 & $38,5-$ \\
60 a 79 & 1,013 & $37,9-$ \\
$\geq 80$ años & 293 & $10,9-$ \\
Total & 2,670 & $100-$ \\
\hline
\end{tabular}

\begin{tabular}{lrr}
\hline Comorbilidades & & \\
\hline Ninguna o no reporta & 1,725 & $64,6-$ \\
1 & 546 & $20,4-$ \\
2 & 277 & $10,4-$ \\
$>2$ & 100 & $3,7-$ \\
\hline
\end{tabular}

\section{Descripción de comorbilidades}

\begin{tabular}{|c|c|c|c|}
\hline$>60$ años & 1,306 & 48,9 & - \\
\hline Hipertensión arterial & 455 & 17,0 & - \\
\hline Diabetes mellitus & 391 & 14,6 & - \\
\hline Insuficiencia renal crónica & 163 & 6,1 & - \\
\hline Obesidad o sobrepeso $^{+}$ & 126 & 4,7 & - \\
\hline Cardiopatías y trastornos del ritmo ${ }^{\ddagger}$ & 58 & 2,7 & - \\
\hline Neumopatías ${ }^{\S}$ & 53 & 1,9 & - \\
\hline Neoplasiasl & 44 & 1,6 & - \\
\hline Cirrosis hepática & 25 & 0,9 & - \\
\hline Colagenopatías" & 18 & 0,7 & - \\
\hline Secuelas de accidente cerebrovascular ${ }^{\beta}$ & 18 & 0,7 & - \\
\hline Patología tiroidea o paratiroidea" & 18 & 0,7 & - \\
\hline PVVS & 5 & 0,2 & - \\
\hline Otros & 8 & 0,3 & - \\
\hline Mortalidad general & 1,234 & 46,2 & - \\
\hline Mortalidad en hospitalización & 1,074 & 87,0 & (1074 de 1234 fallecidos) \\
\hline Mortalidad en hombres & 862 & 69,8 & - \\
\hline Edad entre 40 a 59 años & 626 & 50,7 & (626 de 1234 casos) \\
\hline Hipertensión arterial & 352 & 28,5 & - \\
\hline Diabetes mellitus & 304 & 24,6 & - \\
\hline
\end{tabular}

\section{Mortalidad específica}

\begin{tabular}{lrr}
\hline Cirrosis hepática & 23 de 25 & $92-$ \\
Neoplasias & 36 de 44 & 81,8 - \\
Diabetes mellitus & 304 de 391 & $77,7-$ \\
Hipertensión arterial & 352 de 455 & $77,4-$ \\
Cardiopatía & 44 de 58 & 76,0 - \\
Insuficiencia renal crónica & 117 de 163 & 71,8 -
\end{tabular}

Fuente: departamentos de Actuariado y Estadística y Epidemiología; productividad de Consulta Externa y de áreas de atención CIE - 10 U072. Diagnosticado clínicamente y epidemiológicamente con COVID-19. El ISSS designó hospitales para manejo exclusivo de pacientes con COVID o sospecha, pero debido a la demanda de servicio se dispusieron de otras áreas que fueron adecuadas para tal fin, pero no fueron camas censables. †Diagnóstico según fuente de información. ‡ Cardiopatía y cardiomiopatía isquémica, hipertrófica y dilatada, flutter y aleteo auricular, arritmia cardiaca no especificada. § Enfermedad pulmonar obstructiva o restrictiva crónica, secuelas de tuberculosis y asma bronquial. |Adenocarcinoma tiroideo, cáncer gástrico, tiroides, útero, cérvix, mama, pulmonar, cerebral, próstata, linfomas, leucemias. \Lupus eritematoso sistémico, artritis reumatoide seropositiva, síndrome de sjögren. $\beta$ Sin especificar si es isquémico o hemorrágico. 1 Hipotiroidismo e hipertiroidismo, hiperparatiroidismo posquirúrgico. PWV: persona viviendo con virus de inmunodeficiencia adquirida. 
El análisis de supervivencia presentado según las variables de mayor importancia se presenta en la Tabla 2 y Figura 2. Los datos se presentan distribuidos cada 5 días (del día 1 al 15), la proporción de supervivencia promedio y sus índices de confianza del 95\% se citan en la primera fila. La supervivencia general encontrada fue del $93,6 \%$ al día 1 con IC95 \%, entre $92,5 \%-94,5 \%$ y del $50,4 \%$ (IC95\% 48,1 \%-52,6\%) al día 15, según se encontrara presente o no la condición de interés en estudio. Los pacientes menores de 60 años tuvieron una supervivencia a 15 días del $67 \%$ contra $33,9 \%$ en aquellos con edades mayores. Los que no reportaban presencia de alguna enfermedad crónica presentaron una supervivencia del 66,3\% comparados con aquellos que sí las adolecían (22,5\%). La supervivencia en los no diabéticos es de 55,7 $\%$ y en los diabéticos de $20,9 \%$, en las personas que no tienen hipertensión arterial es de $57,2 \%$ y en los hipertensos de $19,7 \%$. Los que tienen una función renal normal tienen una supervivencia de $52,2 \%$, y en los que tienen nefropatías crónicas como insuficiencia renal crónica la supervivencia es de 22,8 \%. Los que tienen una función cardiaca normal tienen una supervivencia de $51 \%$ en comparación con los cardiópatas crónicos (13,6\%). La supervivencia de los pacientes sin cáncer es de $51,1 \%$ y en los que adolecen de alguna neoplasia es de 3,9\%. En los que tienen un peso normal, la supervivencia es de 51,5\% y los que tienen sobrepeso $u$ obesidad tienen una supervivencia del $26,9 \%$. Las personas sin enfermedades pulmonares tienen una supervivencia de 50,9\%, las personas con neumopatías crónicas de tipo obstructiva tienen una supervivencia de $23,5 \%$, las que tienen una función hepática normal tienen una supervivencia de 50,7 \% y en los cirróticos es de $17,4 \%$. Para todos los casos la prueba de Mantel Cox devolvió un valor de «p» $<0,05$.

\section{Discusión}

El estudio presenta el primer análisis de la probabilidad de muerte por infección pulmonar aguda por COVID-19 en la población de derecho habientes del ISSS y expone las características clínicas y factores de riesgo de muerte. La investigación logró identificar ciertos factores independientes de riesgo incrementado o no de muerte por COVID-19. Algunas condiciones coexistentes como la edad avanzada y sobrepeso u obesidad redujeron la supervivencia a 15 días posterior a su admisión hospitalaria, igual que para condiciones clínicas como diabetes mellitus, hipertensión arterial, insuficiencia renal crónica, cáncer, enfermedades cardiacas y pulmonares crónicas y cirrosis hepática. Una edad menor de 65 años y no adolecer de ninguna de las condiciones patológicas anteriores demostraron tener un efecto contrario.

Las características clínicas de edad, sexo y condiciones clínicas encontradas coinciden con reportes de poblaciones en China, España, Estados Unidos, Perú, Bolivia e Italia y también con lo que informaron varios metaanálisis revisados. El COVID-19 se encontró predominantemente en hombres, similar a lo mismo encontrado en estudios a escala mundia|9,11,13-15,17-22,25,26,29. Esta condición ya ha sido estudiada y parece obedecer a que, aparentemente, los hombres presentan concentraciones plasmáticas más altas de ACE2 que las mujeres y eso puede determinar la mayor predisposición a adquirir la enfermedad.

Los desenlaces fatales se observaron más en jóvenes, pues en su mayoría los pacientes fallecieron a una edad menor que la reportada en otros países, la cual es mayor o igual a 55 años6, ${ }^{9-12,14-16,18,21,22,27,34-36}$. Algunos pacientes tenían una condición de salud coexistente, principalmente diabetes e hipertensión arterial, al igual que lo encontrado en otros estudios 5,78,12,13,17-20. Esta podría ser una explicación del porque la mortalidad es mayor en hombres, ya que una buena cantidad eran hipertensos en tratamiento y con mayores concentraciones de ACE2 plasmáticas.

Una edad mayor de 65 años con condiciones de salud que mantienen un estado inflamatorio crónico como obesidad, secuelas de accidente cerebro vascular, hipertensión arterial, diabetes mellitus, insuficiencia renal crónica, neumo o cardiopatías, son factores que demostraron estar relacionados con una mayor mortalidad por COVID-19, similar a las conclusiones a las que han Ilegado otras investigaciones ${ }^{5-7,8,12,16-20,24,27,30}$.

Un cuarto de los pacientes con COVID-19 concomitantemente eran diabéticos, similar a las poblaciones estudiadas en Estados Unidos $^{16}$, y otros metaanálisis relacionados ${ }^{25}$. La proporción de pacientes con hipertensión arterial es una de las más bajas y solo en algunos estudios llevados a cabo en China ${ }^{1,5,25}$ se reportaron cifras menores, con lo cual queda demostrado como esta condición de salud desempeña un rol importante en la sobrevida a la COVID-19. Otro parámetro distinto en los pacientes de este estudio fue la prevalencia de personas con la insuficiencia renal crónica. Las casuísticas en estudios de España ${ }^{14,32}$, Estados Unidos ${ }^{15,16}$ y China ${ }^{27}$ superaron nuestra casuística, similar a lo encontrado en el caso de la hipertensión arterial.

La población de este estudio presentó una proporción de antecedentes de enfermedad pulmonar obstructiva crónica mayor a la que informaron otros países, entre ellos 


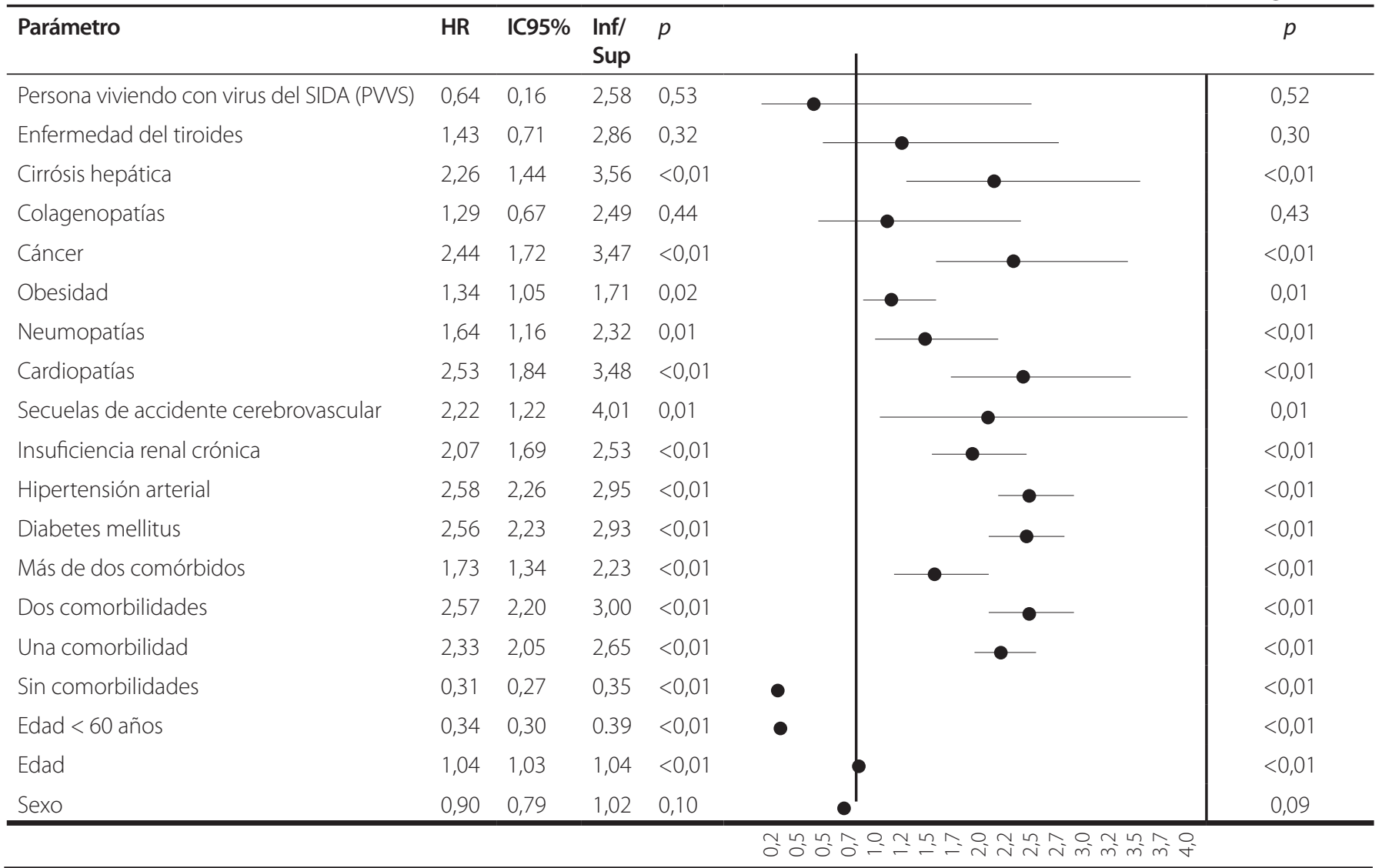

\section{Hazar ratio (IC95\%)}

Protección Incremento de riesgo (muerte)

Figura 1. Análisis multivariado de Cox [Hazard ratio (HR), IC95\% Inf-Sup, valor «p»] y Mantel Cox de mortalidad y parámetros clínicos en pacientes con infección respiratoria aguda por COVID-19.

Tabla 2. Proporciones de supervivencia con intervalos de confianza al $95 \%$ de condiciones clínicas relacionadas a una mayor mortalidad de pacientes con infección respiratoria aguda por COVID-19.

\begin{tabular}{lcccc}
\hline & \multicolumn{4}{c}{ Porcentaje de supervivencia en días - IC95\% (Inf-Sup) } \\
\hline Variable (Log Rank) & 1 & 5 & 10 & 15 \\
\hline Población de pacientes & $93,6(92,5-94,5)$ & $70,8(68,9-72,6)$ & $58,7(56,6-60,8)$ & $50,4(48,1-52,6)$ \\
Edad > 60 años $(<0,01)$ & $94,0(91,7-95,1)$ & $75,4(72,1-78,4)$ & $58,8(54,5-62,9)$ & $33,9(31,0-36,9)$ \\
$\begin{array}{l}\text { Presentar algún comórbido } \\
\text { (<0,01) }\end{array}$ & $89,0(86,7-90,9)$ & $53,6(50,1-56,9)$ & $35,1(31,7-38,5)$ & $22,5(19,4-25,8)$ \\
Diabetes mellitus & & & \\
(<0,01) & $87,9(84,1-90,9)$ & $48,2(42,8-53,4)$ & $34,1(29,0-39,3)$ & $20,9(16,3-25,8)$ \\
Hipertensión arterial & & & & \\
$(<0,01)$ & $89,2(85,8-91,8)$ & $48,6(43,6-53,4)$ & $30,7(26,1-35,4)$ & $19,7(15,6-24,1)$ \\
Fallo renal crónico & & & & \\
$(<0,01)$ & $88,7(82,4-92,8)$ & $51,7(43,2-59,6)$ & $30,9(22,9-39,2)$ & $22,8(15,4-31,1)$ \\
Cardiopatías (0,01) & & & \\
Cáncer (<0,01) & $81,1(67,8-89,4)$ & $41,2(27,2-54,6)$ & $30,5(17,6-44,4)$ & $13,6(4,6-27,2)$ \\
Obesidad (0,02) & $89,7(74,9-96,0)$ & $57,1(39,8-71,1)$ & $23,6(10,9-39,0)$ & $3,9(0,3-16,4)$ \\
Neumopatías (0,01) & $95,0(89,2-97,7)$ & $72,0(66,1-81,9)$ & $54,2(44,3-63,2)$ & $26,9(17,0-37,9)$ \\
Cirrosis hepática $(<0,01)$ & $94,0(82,5-98,0)$ & $69,9(54,5-81,1)$ & $38,6(24,2-52,7)$ & $23,4(11,2-38,4)$ \\
\hline
\end{tabular}


A supervivencia general

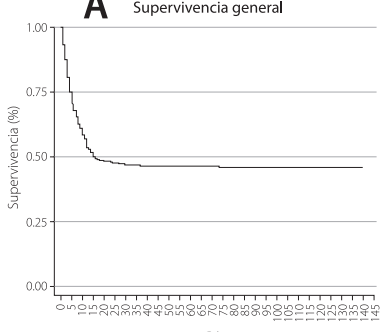

Días

D Diabetes mellitus
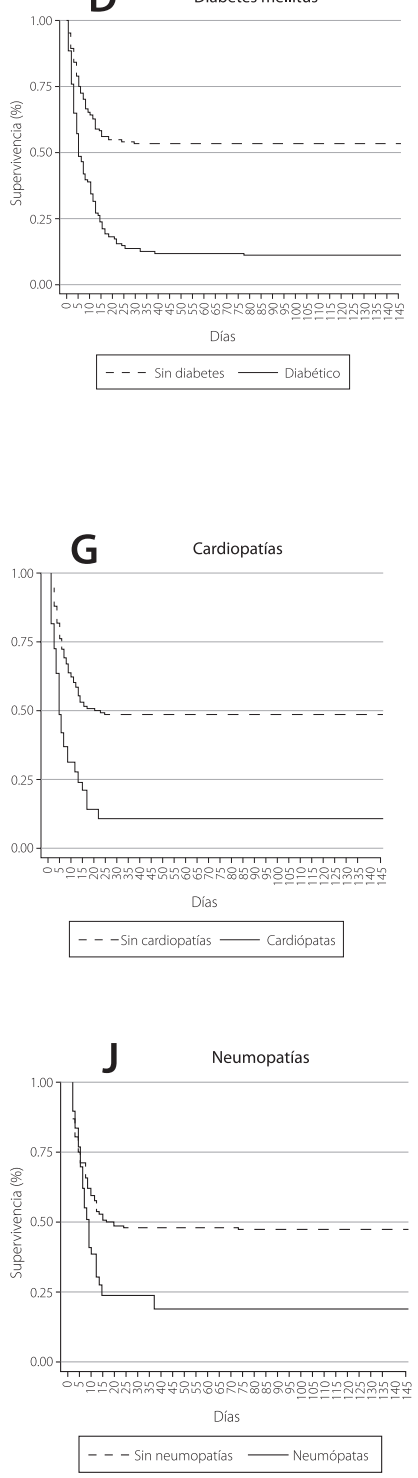

B

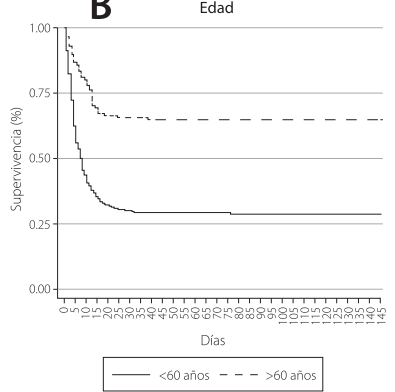

E Hipertensión arterial

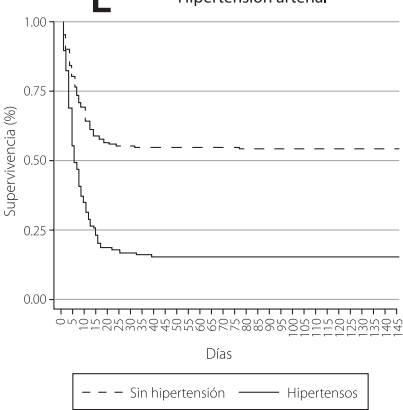

--- Sin hipertensión — Hipertensos
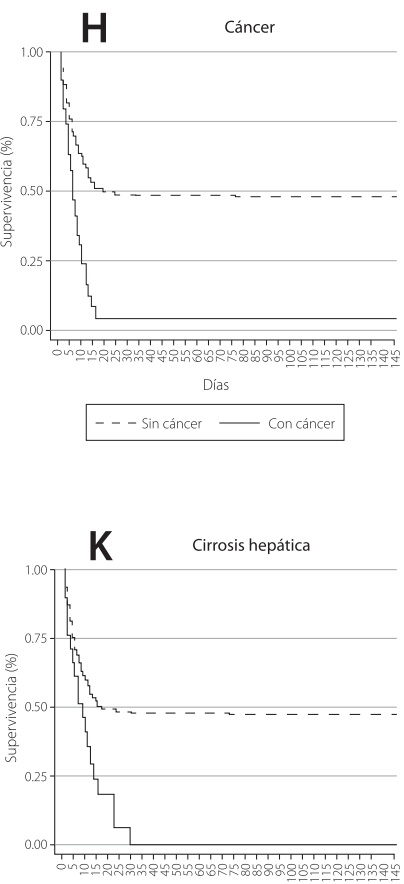

Días

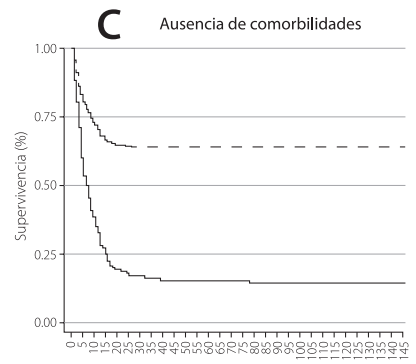

Con comorbidos --- sin comorbidos
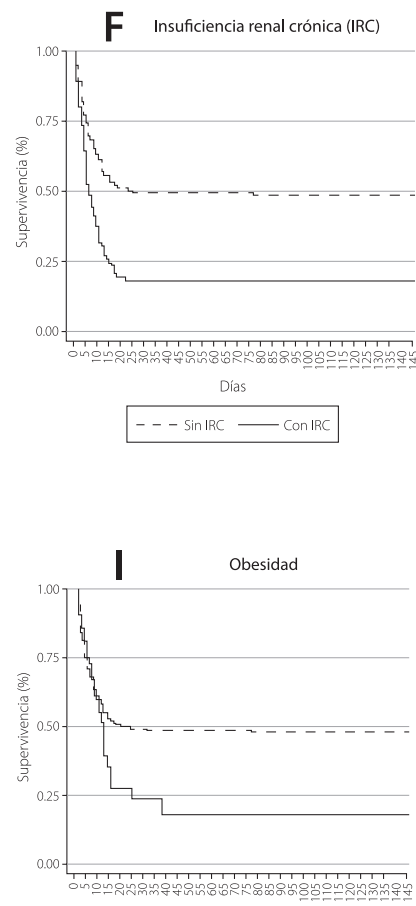

- - - Peso normal - obesos

Figura 2. Curvas de Kaplan Meier de los diferentes factores pronósticos según (A) sobrevivencia general, (B) edad, (C) ausencia de enfermedades, (D) diabetes, (E) hipertensión, (F) IRC, (G) cardiopatías, (H) cáncer, (I) obesidad, (J) neumopatías, (K) cirrosis, todos con una $\mathrm{p}<0,05$.

China ${ }^{5,7,8,17}$, y respecto a las cardiopatías crónicas, nuestra casuística es similar a la del Perú18, constituyéndose en una de las menores proporciones que se encontraron en investigaciones consultadas.

Para los pacientes con secuelas de accidente cerebro vascular con infección pulmonar por COVID-19, no se logró encontrar tampoco una casuística menor a la de las personas de esta investigación. En el caso de otras enfermedades crónicas como cáncer, obesidad y cirrosis hepática, los hallazgos en estos pacientes no difieren de manera importante a los resultados de otros estudios.

Las condiciones de salud encontradas en los pacientes de este estudio y que están 
relacionadas con un mayor riesgo de morir por COVID-19 se asemejan mucho con otras poblaciones en China ${ }^{17,20,29,30}$ y Bolivia ${ }^{19}$, en las cuales se logró demostrar que la diabetes, hipertensión, insuficiencia renal, cáncer, entre otras, son condiciones de salud que están relacionadas con una probabilidad incrementada de muerte por COVID-19. Una edad avanzada incrementa las fatalidades hasta en 12 veces ${ }^{13,15,17,18} \mathrm{y}$, para nuestros pacientes mayores de 60 años, esta posibilidad se incrementó en 1,04 veces por cada año de vida.

Las secuelas de accidente cerebrovascular llegaron a tener un riesgo de hasta 4 veces mayor de mortalidad, muy similar a otros estudios ${ }^{11,27}$, lo cual pudiera estar relacionado a la incapacidad de las personas con esta condición de poder expresar que están enfermas y al detectarlo puede que ya fuera demasiado tarde. Los pacientes con hipertensión arterial también vieron incrementada su mortalidad al adquirir la enfermedad $^{19}$, dato que coincidió con los de este estudio y están en sintonía con resultados de investigaciones en España que registraron una mortalidad casi tres veces mayor ${ }^{14,32}$. Una condición de fatalidad similar fue encontrada en personas con cardiopatías crónicas e infección por COVID-1999-11,14,23, las cuales, según estudios, en algunos hospitales de China hubo un incremento en las muertes hasta de 4 veces más ${ }^{10,27}$. La diabetes mellitus también presentó un efecto negativo en la sobrevida de las personas como lo encontrado en series de China ${ }^{9}$ y Bolivia ${ }^{19}$. La anterior observación es también válida para los casos de pacientes con cáncer ${ }^{16,22}$, insuficiencia renal crónica ${ }^{14,16,32}$, enfermedad pulmonar obstructiva crónica ${ }^{14,22,}$ y cirrosis hepática ${ }^{14}$

El trabajar únicamente con información proveniente de los registros de hospitalización y no disponer de datos del resto de sistemas prestadores de servicios de salud del país es una de las mayores limitantes de este estudio, debido a que otras condiciones de interés pudieron quedar fuera del análisis. Debido a lo anterior los datos obtenidos en este estudio pudieran variar al integrar otra información de los expedientes clínicos y del resto de instituciones de salud, por lo cual se recomienda considerar estos resultados con cautela.

Es necesario realizar otros análisis que incluyan parámetros clínicos, detalles de la evolución clínica, conocer el estado pulmonar de los pacientes al momento del ingreso y su relación con el pronóstico, la respuesta a los distintos esquemas de manejo terapéuticos, de soporte ventilatorio, maniobras de ventilación prona y cuando se comience a vacunar a la población, evaluar el impacto de esta estrategia en la casuística para conocer más acerca de esta enfermedad en nuestro país.

\section{Conclusión}

Las enfermedades crónicas que como parte de su evolución alteran los mecanismos de defensa y mantienen un estado pro inflamatorio crónico se asociaron con una mayor mortalidad. Tener menos de 60 años y no adolecer de ninguna de estas enfermedades crónico degenerativas se relaciona con un riesgo menor de muerte por COVID-19 y una supervivencia mayor, mientras que la diabetes mellitus, hipertensión arterial, insuficiencia renal crónica, enfermedades cardiacas, pulmonares crónicas, obesidad, cáncer y cirrosis hepática se relacionan con una mayor mortalidad y menor sobrevida. No se logró demostrar una relación entre el sexo de los pacientes y las patologías del tiroides y paratiroides ni con las colagenopatías, lupus eritematoso sistémico y artritis reumatoide seropositiva con la mortalidad por COVID.

\section{Referencias bibliográficas}

1. Centros para el Control y la Prevención de Enfermedades, Centro Nacional de Inmunización y Enfermedades Respiratorias(NCIRD). Historia de la pandemia de gripe de 1918. (2018). Consultado en mayo 2021. Disponible en: https://www.cdc.gov/flu/pandemicresources/1918-commemoration/1918pandemic-history.htm

2. Lu R, Zhao X, Li J, et al., Genomic characterization and epidemiology of 2019 novel coronavirus: implications for virus origins and receptor binding. Lancet 2020; 395:565-74. DOI: 10.1016/S01406736(20)30251-8

3. Zhu N, Zhang D, Wang W, et al. A Novel Coronavirus from Patients with Pneumonia in China, 2019. N Engl J Med 2020; 382:727733, DOI: 10.1056/NEJMoa2001017

4. World Health Organization. Coronavirus disease (COVID-19) situation reports. Consultado el 30 mayo 2020. Disponible en https://www.who.int/emergencies/diseases/ novel-coronavirus-2019/situation-reports/

5. Guan W, Ni Z, Yu Hu W, et al. Clinical characteristics of coronavirus disease 2019 in China, N Engl J Med 2020;382:1708-20. DOI: 10.1056/NEJMoa2002032

6. Zhou F, Yu T, Du R, et al. Clinical course and risk factors for mortality of adult inpatients with COVID-19 in Wuhan, China: 
a retrospective cohort study. Lancet. 2020 Mar 28;395(10229):1054-1062. DOI: 10.1016/ S0140-6736(20)30566-3.

7. Guan W, Liang W, Zhao Y, et al. Comorbidity and its impact on 1590 patients with COVID-19 in China: a nationwide analysis. Eur Respir J 2020; 55: 2000547. DOI: 10.1183/13993003.00547-2020

8. López A, Domínguez R. Medidas de contingencia ante el COVID-19 en El Salvador, Alerta. 2021;4(1):78-79. DOI 10.5377/alerta.v4i1.10762

9. Gobierno de El Salvador. COVID-19 Reporte Diario. Gobierno de la Republica de El Salvador. 2021. Consultado el 21 de enero de 2021. Disponible en https://covid19.gob. sv/diarios/

10. Oliva Marín JE. COVID-19 en la niñez y adolescencia. Alerta. 2021;4 (1):49-61. DOI 10.5377/alerta.v4i1.9780

11. Hui Du, Rong L, Qing C, et al. Predictors of mortality for patients with COVID-19 pneumonia caused by SARSCOV-2: a prospective cohort study. Eur Respir J 2020; 55: 2000524. DOI:10.1183/13993003.005242020

12. Yang $K$, Sheng $Y$, Huang $C$, et al. Clinical characteristics, outcomes, and risk factors for mortality in patients with cancer and COVID-19 in Hubei, China: a multicenter, retrospective, cohort study. Lancet Oncol 2020; 21: 904-13. DOI:10.1016/ S14702045(20)30310-7

13. Gil-Rodrigo A, Miró O, Piñera P, et al. Evaluación de las características clínicas y evolución de pacientes con COVID-19 a partir de una serie de 1000 pacientes atendidos en servicios de urgencias españoles. Emergencias: revista de la Sociedad Española de Medicina de Emergencias, 32(4), 233-241

14. Argenziano M, Bruce $\mathrm{S}$, Slater $\mathrm{CL}$, et al. Characterization and clinical course of 1000 patients with coronavirus disease 2019 in New York: retrospective case series. BMJ 2020;369:m1996. DOI: 10.1136/bmj.m1996

15. Suleyman G, Fadel RA, Malette KM, et al. Clinical Characteristics and Morbidity Associated with Coronavirus Disease 2019 in a Series of Patients in Metropolitan Detroit. JAMA Network Open. 2020;3(6): e2012270. DOI:10.1001/jamanetworkopen.2020.12270

16. Liang W, Guan W, Li C, et al. Clinical characteristics and outcomes of hospitalized patients with COVID-19 treated in Hubei (epicenter) and outside Hubei (nonepicenter): a nationwide analysis of China. Eur Respir J 2020; 55: 2000562 DOI:10.1183/13993003.00562-2020].

17. Mejía F, Medina C, Cornejo E., el al. Características clínicas y factores asociados a mortalidad en pacientes adultos hospitalizados por COVID-19 en un hospital público de Lima, Perú. DOI: $10.1590 /$ $\underline{\text { SciELOPreprints.858 }}$

18. Escalera J, Lizon N, Maldonado A., et al (2020). Risk factors for mortality in patients with Coronavirus Disease 2019 (COVID-19) in Bolivia: An analysis of the first 107 confirmed cases. Le Infezioni in Medicina, 28(2), 238-242. Disponible en https://www. researchgate.net/publication/341569077

19. Chen T, Dai Z, Mo P, et al. Clinical Characteristics and Outcomes of Older Patients with Coronavirus Disease 2019 (COVID-19) in Wuhan, China: A SingleCentered, Retrospective Study. J Gerontol A Biol Sci Med Sci, 2020, (75), 9, 1788-1795. DOl:10.1093/gerona/glaa089

20. Lavarone M, DiAmbrossio R, Soria A, et al. High rates of 30-day mortality in patients with cirrhosis and COVID-19. Hepatol. 2020,1-9. DOI:10.1016/j.jhep.2020.06.001

21. Dai M, Lui D, Lui M, et al. Patients with Cancer Appear More Vulnerable to SARSCoV-2: A Multicenter Study during the COVID-19 Outbreak. Cancer Discov June 1 2020 (10) (6) 783-791; DOI: 10.1158/21598290.CD-20-0422.

22. Robilotti, E.V., Babady, N.E., Mead, P.A. et al. Determinants of COVID-19 disease severity in patients with cancer. Nat Med 26, 1218-1223 (2020). DOI: 10.1038/s41591020-0979-0

23. Garg S, Kim L, Whitaker M, et al. Hospitalization Rates and Characteristics of Patients Hospitalized with LaboratoryConfirmed Coronavirus Disease 2019 — COVID-NET, 14 States, March 1-30, 2020. MMWR Morb Mortal Wkly Rep 2020; 69:458-464. DOI: 10.15585/mmwr. mm6915e3external icon.

24. Yang J, Zheng Y, Gou X, et al. Prevalence of comorbidities and its effects in patients infected with SARS-CoV-2: a systematic review and meta-analysis. Int J Infect Dis. 2020; 94:91-95. DOl: 10.1016/j. ijid.2020.03.017

25. Escobar G, Matta J, Ayala, et al. Características clínico epidemiológicas de pacientes fallecidos por covid-19 en un hospital nacional de Lima, Perú. Rev. Fac. Med. Hum. DOI: 10.25176/rfmh.v20i2.2940

26. Chen $R$, Liang $W$, Jiang $M$, el al., Risk Factors of Fatal Outcome in Hospitalized Subjects with Coronavirus Disease 2019 From a Nationwide Analysis in China. CHEST 2020, 158(1)97-105. DOl: 10.1016/j. chest.2020.04.010

27. G-Q Qian, N-B Yang, F Ding, et al, Epidemiologic and clinical characteristics of 91 hospitalized patients with COVID-19 in Zhejiang, China: a retrospective, multicenter case series, QJM: An International Journal of Medicine, (113), 7, 474-481. DOl: 10.1093/qjmed/hcaa089 
28. Tie $L$ Chen, $M$, Zhe $D$, Pingzheng $M$, et al, Clinical Characteristics and Outcomes of Older Patients with Coronavirus Disease 2019 (COVID-19) in Wuhan, China: A SingleCentered, Retrospective Study, J Gerontol: Series A, Volume 75, Issue 9, 1 September 2020, Pages 1788-1795. DOl: 10.1093/ gerona/glaa089

29. Ruan, Q., Yang, K., Wang, W. et al. Clinical predictors of mortality due to COVID-19 based on an analysis of data of 150 patients from Wuhan, China. Intensive Care Med 46, 846-848 (2020). DOl:10.1007/s00134-02005991-X

30. Emani A, Javanmardi F, Pirbonyeh N, Akbari A. Prevalence of underlying diseases in hospitalized patients with COVID-19: a systematic review and meta-analysis. Arch Acad Emerg Med. 2020;(1): e35. Disponible en https://www.ncbi.n/m.nih.gov/pmc/ articles/PMC7096724/

31. Gutiérrez J, Montero J, Jiménez F, et al. Variables asociadas con mortalidad en una población de pacientes mayores de 80 años y con algún grado de dependencia funcional, hospitalizados por COVID-19 en un Servicio de Geriatría. Rev Esp Geriatr Gerontol. 2020; S0211-139X (20)30109-8. DOl: 10.1016/j.regg.2020.07.002
32. Kumar, A., Arora, A., Sharma, P., Anikhindi, S. A., Bansal, N., Singla, V., Khare, S., \& Srivastava, A. (2020). Is diabetes mellitus associated with mortality and severity of COVID-19? A meta-analysis. Diabetes Metab Res. Rev 2020 July-August; 14(4): 535-545. DOI: 10.1016/j. dsx.2020.04.044

33. Guo, W., Li, M., Dong, Y., et al. (2020). Diabetes is a risk factor for the progression and prognosis of COVID-19. Diabetes Metab Res. Rev. DOI: 10.1002/dmrr.3319

34. Guo T, Fan Y, Chen M, et al. Cardiovascular implications of fatal outcomes of patients with coronavirus disease 2019 (COVID-19). JAMA cardiol.2020;(5(7):811-818. DOI: :10.1001/jamacardio.2020.1017

35. Aggarwal G, Lippi G, Michael Henry B. Cerebrovascular disease is associated with an increased disease severity in patients with Coronavirus Disease 2019 (COVID-19): A pooled analysis of published literature. Stroke. 2020;15(4):385-389. DOI:10.1177/1747493020921664

36. Huang C, Wang Y, Li X, et al. Clinical features of patients infected with 2019 novel coronavirus in Wuhan, China. Lancet. 2020 feb 15;395(10223):497-506. DOI: 10.1016/ s0140-6736(20)30183-5 